\title{
A Boundary Element Method iterative procedure to compute the compressible response of an airfoil subjected to incoming turbulence
}

\author{
Leandro D. Santana*, Christophe Schram ${ }^{\dagger}$ \\ von Karman Institute for Fluid Dynamics, Sint-Genesius-Rode, Belgium \\ Wim Desmet $\ddagger$ \\ KU Leuven, Leuven, Belgium
}

\begin{abstract}
This paper proposes an innovative numerical technique for computing the aeroacoustic response function of an airfoil subjected to incoming turbulence, accounting for trailingedge back-scattering effects and valid in the high-frequency subsonic compressible regime. The methodology is based on the linearized potential flow equations, rewritten as the Helmholtz equation, and solved using a Boundary Element Method (BEM) solver. This approach permits to account for some realistic airfoil geometry effect. It has been validated against Amiet theory for non-compact incident wavenumbers, showing good agreement for both direct propagation and back-scattering correction. Finally, this methodology is applied to a NACA-0012 wake-airfoil interaction case with wake parameters obtained from experimental data, showing trends in good agreement with literature.
\end{abstract}

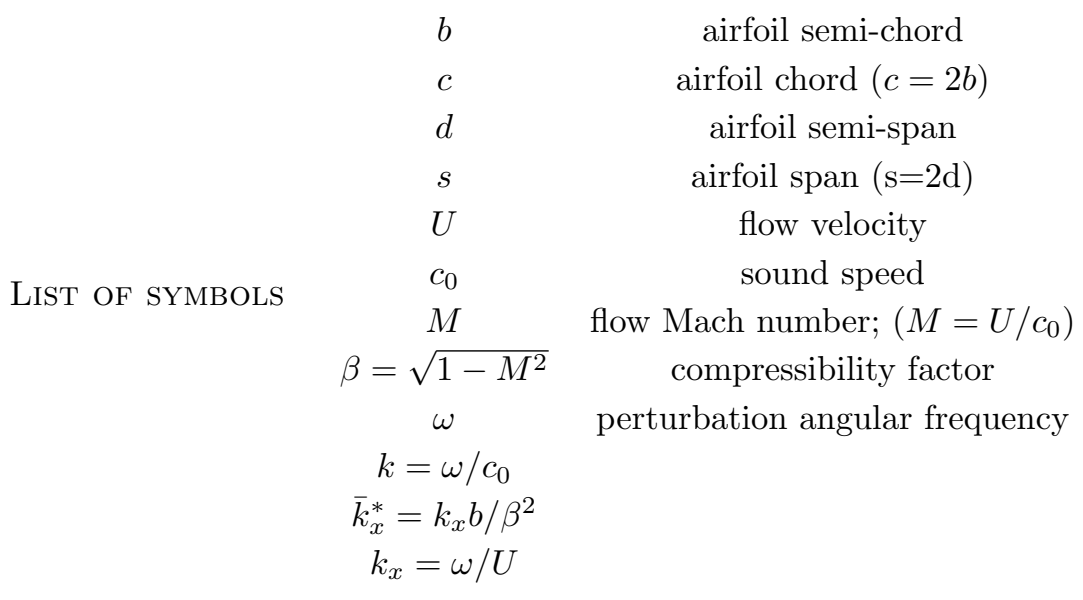

\section{Introduction}

In turbomachinery applications such as contra-rotating-open-rotors (CRORs) or rotor-stator cascades, important tonal and broadband noise is produced as a result of intense force fluctuations associated with potential or viscous interactions. In particular, viscous wake-airfoil interaction noise represents an important source mechanism, having been the focus of many theoretical, numerical and experimental investigations reported in the literature. ${ }^{1-10}$ Semi-analytical predictive approaches are essential for the conceptual and preliminary design phases of rotating machines, permitting quick noise assessments and optimization studies.

${ }^{*} \mathrm{PhD}$ candidate, Aeronautics and Aerospace Department, Waterloosesteenweg 72, B-1640 Sint-Genesius-Rode, AIAA Student Member.

${ }^{\dagger}$ Associate Professor, Aeronautics and Aerospace Department, Waterloosesteenweg 72, B-1640 Sint-Genesius-Rode, AIAA Member.

${ }^{\ddagger}$ Full professor, Department of Mechanical Engineering, Celestijnenlaan 300, B-3001 Heverlee. 
The development of such tools, based on a deep understanding of the physics, has been challenging authors for several years. ${ }^{11-15}$ While semi-analytical approaches offer the clear advantage of fast evaluation, they generally impose drastic simplifications such as small thickness, camber and angle-of-attack, and assimilate the airfoil to a large aspect ratio rectangular flat plate subjected to uniform incoming flow. In an attempt to relax these assumptions and tackle more realistic blade geometries, researchers have proposed segmentation methods in order to account for spanwise-varying incoming turbulence properties, ${ }^{16}$ non-rectangular strip shape, ${ }^{17}$ and the alteration of the incoming turbulence spectrum due to leading edge thickness. ${ }^{18}$

In order to account for more complex incoming flows and real airfoil geometry effects, numerical methodologies have been proposed for the flow solution. ${ }^{19,20}$ Glegg and Devenport ${ }^{15}$ used a the panel method to predict turbulence-airfoil interaction noise, and obtained a good agreement with Amiet's theory for $\mu=\bar{k}_{x}^{*} M$, within $1 \mathrm{~dB}$ for $\mu \leq 3.5$ and within $2 \mathrm{~dB}$ for $\mu \leq 5.3$, respectively. But this technique is not suited to predict the noise for higher frequencies, since it solves the potential flow equation using a Laplace formulation, only valid in the incompressible asymptotic limit, just as in the pioneering work of von Kármán and Sears ${ }^{21}$ where the airfoil response to a periodic perturbation was computed for a potential incompressible flow. Amongst the theoretical works aimed at addressing compressibility effects $\left({ }^{22-25}\right)$, Amiet ${ }^{26}$ presented a successful formulation where the airfoil response to an high-frequency incoming periodic perturbation is computed by the solution of the Helmholtz equation using the Schwarzschild technique. ${ }^{27}$ This analytical approach, further developed by Roger and Moreau ${ }^{28}$ as an iterative procedure to take into account leading-trailing edges backscattering effects in finite chord length airfoils, has inspired the present work. Indeed, the same iterative scheme is applied in this paper, but implementing a numerical determination of the airfoil response at each iteration, solving the compressible Helmholtz equation by means of a Boundary Element Method (BEM) discretization. It will be shown that the proposed methodology predicts both realistic airfoil geometry effects and compressibility effects.

In what follows, we remind how the small perturbation compressible potential flow equations can be rewritten in the form of the Helmholtz equation, and detail the boundary conditions of the aerodynamic problem. The analogy that exists between the aerodynamic and the acoustic BEM variables is introduced and the proposed technique is validated against Amiet's theory. The method is then applied to a wake-airfoil interaction problem, using wake parameters obtained by the present authors in a previous experimental study. $^{29}$ This profile is set as input for the present methodology for the computation of the aerodynamic response and far-field noise of a flat plate and airfoils with thickness of $1 \%, 6 \%$ and $12 \%$.

\section{Problem formulation}

\section{A. The boundary value problem}

Following Amiet's formalism, ${ }^{26}$ we consider an airfoil lying in the $z=0$ plane between $0 \leq x / b \leq 2$ with chord $c=2 b$, span $s=2 d$ placed in a mean flow with velocity $U$, as sketched in Fig. 1 .

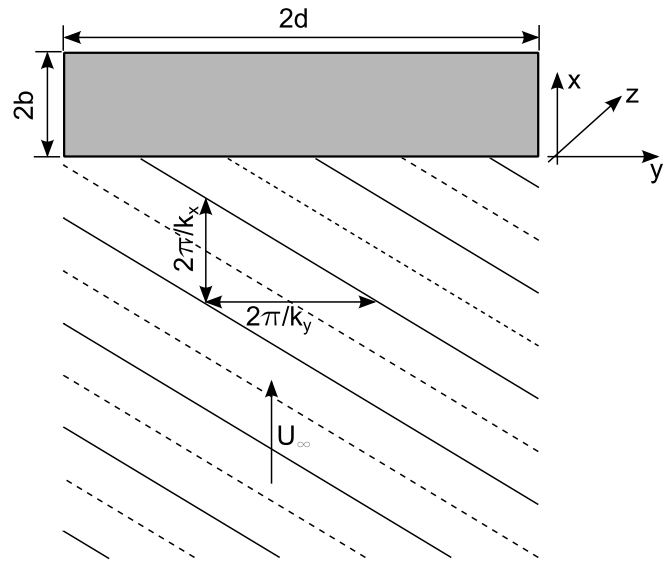

Figure 1. Airfoil-gust interaction scheme.

The linearized airfoil theory defines a boundary value problem composed by Eq. 1 and boundary condi- 
tions defined by Eqs. 2, 3 and 4:30

$$
\begin{array}{rrr} 
& {\left[\nabla^{2}-\frac{1}{c_{0}^{2}} \frac{D^{2}}{D t^{2}}\right] \phi(x, y, z)=0} & \\
\phi(x, y, 0, t) & =0 & x \leq 0 \\
\frac{\partial \phi}{\partial z}(x, y, 0, t) & =-w(x) & 0<x \leq 2 b \\
\frac{D \phi}{D t}(x, y, 0, t) & =0 & x>2 b
\end{array}
$$

where $x, y$ and $z$ are directions in a normal system of coordinates and time $t, c_{0}$ is the sound speed. The total derivative operator is defined as:

$$
\frac{D}{D t}=\frac{\partial}{\partial t}+U \frac{\partial}{\partial x}
$$

Eq. 1 is the convected Helmholtz equation. The Eq. 2 boundary condition imposes zero flow potential at the region upstream the airfoil leading edge. The boundary condition of Eq. 3 imposes the non-penetration boundary condition on the airfoil surface and Eq. 4 imposes zero pressure jump on the airfoil wake.

\section{B. Fourier decomposition of the flow potential}

Considering that the flow potential $\phi$ can be defined as a function of three Fourier-type functions as:

$$
\phi(x, y, z)=\varphi(x, z) e^{i \omega t} e^{i \gamma x} e^{i \alpha y}
$$

where $i=\sqrt{-1}, \omega$ is the perturbation angular frequency, $\gamma=\frac{k M}{\beta^{2}}, \alpha=-k_{y}, M$ is the flow Mach number, $k=\omega / c_{0}$ and $\beta=\sqrt{1-M^{2}}$. Replacing Eq. 6 into Eq. 1 we have: ${ }^{31}$

$$
\beta^{2} \frac{\partial^{2} \varphi}{\partial x^{2}}+\left(\frac{k^{2}}{\beta^{2}}-\alpha^{2}\right) \varphi+\frac{\partial^{2} \varphi}{\partial z^{2}}=0
$$

using the following relations for non-dimensionalization:

$$
\bar{x}=\frac{x}{b} \quad, \quad \bar{y}=\frac{\beta y}{b} \quad, \quad \bar{z}=\frac{\beta z}{b} .
$$

Eq. 7 can be rewritten as:

$$
\frac{\partial^{2} \varphi}{\partial \bar{x}^{2}}+\frac{\partial^{2} \varphi}{\partial \bar{z}^{2}}+\frac{b^{2}}{\beta^{2}}\left(\frac{k^{2}}{\beta^{2}}-\alpha^{2}\right) \varphi=0
$$

adopting the following definitions:

$$
k_{x}=\frac{\omega}{U} \quad, \quad k=k_{x} M \quad, \quad \bar{k}_{i}=k_{i} b
$$

Eq. 9 is simplified to:

$$
\frac{\partial^{2} \varphi}{\partial \bar{x}^{2}}+\frac{\partial^{2} \varphi}{\partial \bar{z}^{2}}+\left(\frac{\bar{k}_{x}^{2} M^{2}}{\beta^{4}}-\frac{\bar{k}_{y}^{2}}{\beta^{2}}\right) \varphi=0
$$

which is written as a canonical Helmholtz equation as:

$$
\frac{\partial^{2} \varphi}{\partial \bar{x}^{2}}+\frac{\partial^{2} \varphi}{\partial \bar{z}^{2}}+\kappa^{2} \varphi=0
$$

where

$$
\kappa^{2}=\mu^{2}-\frac{\bar{k}_{y}^{2}}{\beta^{2}} \quad, \quad \mu=\frac{\bar{k}_{x} M}{\beta^{2}}=\bar{k}_{x}^{*} M \quad, \quad \bar{k}_{x}^{*}=\frac{\bar{k}_{x}}{\beta^{2}} .
$$

The nature of the problem differs depending on the sign of $\kappa^{2}$. If $\kappa^{2}>0$ the partial differential equation (PDE) is classified as a hyperbolic equation and the gust is named supercritical. In this kind of problem, an initial perturbation is not seen at the same instant on all positions of the flow, but it is wave-likely 
propagated with constant and finite speed along characteristic lines. In case of $\kappa^{2}<0$, the PDE is elliptic and the gust is said subcritical. Subcritical gusts contribute to the far-field for finite-span airfoils only, as they produce evanescent waves otherwise.

For completeness, as demonstrated by Christophe, ${ }^{31}$ considering that the potential perturbation can be represented by Fourier-type components, the boundary conditions of Eqs. 2,. 3, and. 4 can be rewritten as:

$$
\begin{array}{rlrl}
\varphi(\bar{x}, 0) & =0 & \bar{x} & \leq 0 \\
\frac{\partial \varphi}{\partial \bar{z}}(\bar{x}, 0) & =-\frac{w_{0} b}{\beta} e^{-\bar{k}_{x}^{*} \bar{x}} & 0<\bar{x} \leq 2 \\
\left(i \bar{k}_{x}^{*}+\frac{\partial}{\partial \bar{x}}\right) \varphi(\bar{x}, 0) & =0 & \bar{x}>2
\end{array}
$$

\section{The Schwarzschild procedure}

The boundary value problem described by Eq. 12 and boundary conditions from Eqs. 14, 15 and 16 can be solved using the Schwarzschild ${ }^{27}$ procedure. The Schwarzschild problem states that if a function $\phi$ satisfies the wave equation (Eq. 1) and has boundary conditions which can be written on the form of:

$$
\begin{aligned}
\phi(x, 0) & =F(x) & & x>0 \\
\frac{\partial}{\partial z} \phi(x, 0) & =0 & & x<0
\end{aligned}
$$

the solution is the following:

$$
\phi(x, z)=\frac{1}{\pi} \int_{0}^{\infty} G(x, \xi, z) F(\xi) d \xi
$$

where $G$ is the Green's function solution of the boundary value problem. This function is normally dependent of the geometry of the problem and the perturbation frequency. A numerical approach has to be followed to obtained a tailored Green's function for an arbitrary airfoil geometry, and in this work we use a Boundary Element Method solver to this end.

Since the Schwarzschild solution can satisfy only two boundary conditions (described in Eq. 17 and Eq. 18) Amiet ${ }^{26}$ suggests that a iterative solution procedure where the now flow condition through the airfoil is satisfied together with one of the two other boundary conditions of the problem.

\section{The solution strategy}

Following the Amiet ${ }^{26}$ strategy for computing the airfoil response to a periodic gust, the Schwarzschild procedure could be used to divide the problem into sub-problems, where the non-penetration boundary condition is satisfied together with another boundary condition and superimposed to satisfy the three different boundary conditions.

The first sub-problem consists on solving Eq. 12, subjected to the non-penetration boundary condition along all domain. This problem is represented in Fig. 2.

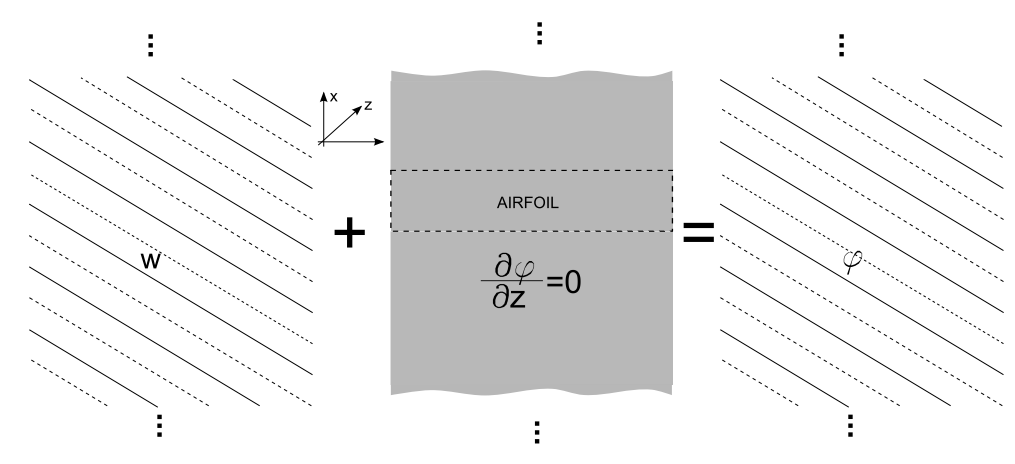

Figure 2. First step: Gust perturbation cancellation scheme. 
Once the flow potential is obtained, the Amiet methodology suggests that the zero potential, at the upstream region of the airfoil, should be satisfied. In consequence Eq. 12 should be solved with the imposition of the Eq. 14 boundary condition and the non-penetration boundary condition on the region downstream the airfoil leading edge. This problem is represented in Fig. 3.

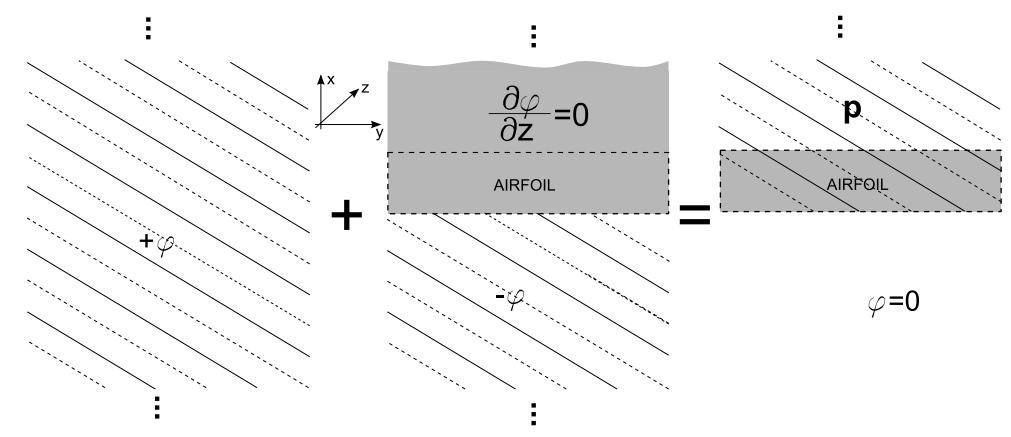

Figure 3. Second step: Leading edge correction scheme.

After imposing the leading-edge boundary condition, to satisfy the Kutta condition and the wake condition, the boundary condition of Eq. 16 is applied for on the region downstream the airfoil trailing edge. This problem is represented in Fig. 4.

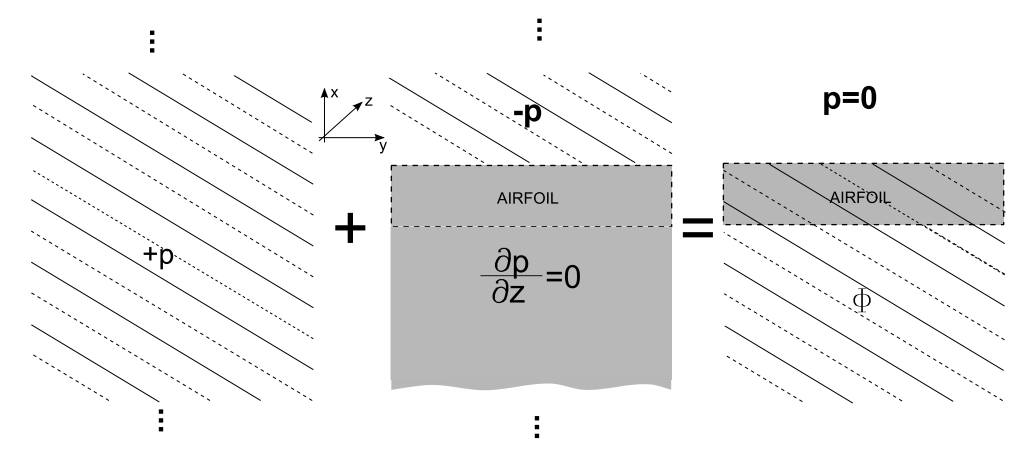

Figure 4. Third step: Trailing edge correction scheme.

Since the non-penetration boundary condition has already been imposed, on the airfoil region of the domain, for three previous steps, this boundary condition is already satisfied. This iterative scheme is shown to be not exact, since, after two iterations, a remainder potential is left at the region upstream the airfoil trailing edge. Roger ${ }^{28}$ shows that this potential can be considered negligible after two iterations.

\section{The BEM approach for solving linearized compressible flow equations}

\section{A. Solving the linearized flow equations using an acoustic solver}

Since this methodology can be applied using a commercial acoustic solver it is necessary to translate the acoustic solver variables into potential flow variables. Our numerical method solves the Helmhotz equation using the pressure perturbation as acoustic variable:

$$
\nabla^{2} p(x, y, z)+k^{2} p(x, y, z)=0
$$

subjected to the following set of possible boundary conditions:

- Imposed pressure: $\bar{p}=p$;

- Imposed normal velocity: $\bar{v}_{n}=\frac{i}{\rho \omega} \frac{\partial p}{\partial n}$; 
- Imposed normal impedance: $p=\frac{i \bar{Z}}{\rho \omega} \frac{\partial p}{\partial n}$

Comparing Eq. 12 and Eq. 20 it is possible to find that both equations are the same if the following set of equalities are considered:

- $\varphi=p$;

- $\kappa^{2}=k^{2}$;

Similarly to the approach adopted for solving the Amiet problem, the present work will use a 3 steps procedure for computing the generic airfoil response to a periodic gust. From Eq. 12, in the first step, the following problem should be solved:

$$
\frac{\partial^{2} \varphi^{(0)}}{\partial x^{2}}+\frac{\partial^{2} \varphi^{(0)}}{\partial z^{2}}+\kappa^{2} \varphi^{(0)}=0
$$

subjected to an incoming gust with profile:

$$
w(x, \omega)=w_{0}(\omega) e^{-i k_{x} x}
$$

It is equivalent to an acoustic BEM problem if the following set of variable substitutions is made:

$$
\begin{aligned}
\varphi^{(0)} & =p \\
\kappa^{2} & =k^{2} \\
w(x, \omega) & =\frac{i}{\rho \omega} \frac{\partial p}{\partial n}
\end{aligned}
$$

where the left hand side of Eqs. 23, 24 and 25 refer to the variables associated with to the aerodynamic problem, while the right hand side variables of these equations refer to the BEM solver variables.

In the second step, the correction potential $\varphi^{(1)}$ should be found in such a way that this potential is zero for $x<0$. In this way, the potential $\psi_{1}$, which satisfies the relation $\varphi^{(1)}=\varphi^{(0)}+\psi_{1}$, should be computed. This step leads to a second Helmholtz equation to be solved:

$$
\frac{\partial^{2} \psi_{1}}{\partial \bar{x}^{2}}+\frac{\partial^{2} \psi_{1}}{\partial \bar{z}^{2}}+\kappa^{2} \psi_{1}=0
$$

subjected to the following boundary conditions:

$$
\begin{aligned}
\psi_{1}(\bar{x}, 0) & =-\varphi^{(0)}(\bar{x}, 0) & & \bar{x} \leq 0 \\
\frac{\partial \psi_{1}}{\partial \bar{z}} & =0 & & x>0
\end{aligned}
$$

This problem is equivalent to an acoustic BEM problem if the following set of variable substitutions is made:

$$
\begin{aligned}
\kappa^{2} & =k^{2} \\
\psi_{1} & =p \\
\varphi^{(0)} & =\bar{p}
\end{aligned}
$$

where the left hand side of Eqs. 29, 30 and 31 refers to the variables associated with the aerodynamic problem, while the right hand side variables refer to the BEM solver variables.

In the third step the trailing edge correction is executed. In this way, the following problem should be solved:

$$
\frac{\partial^{2} P_{2}}{\partial \bar{x}^{2}}+\frac{\partial^{2} P_{2}}{\partial \bar{z}^{2}}+\kappa^{2} P_{2}=0
$$

with the following boundary conditions:

$$
\begin{array}{rlrl}
P_{2}(\bar{x}, 0) & =-P_{1}(\bar{x}, 0) & & \bar{x} \geq 2 \\
\frac{\partial P_{2}}{\partial \bar{z}}(\bar{x}, 0) & =0 & \bar{x}<2
\end{array}
$$


It is equivalent to an acoustic BEM problem if the following set of variable substitutions is made:

$$
\begin{aligned}
& \kappa^{2}=k^{2} \\
& P_{2}=p \\
& P_{1}=\bar{p}
\end{aligned}
$$

where the left hand side of Eqs. 35, 36 and 37 refers to the variables associated with the aerodynamic problem, while, the right hand side variables refer to the BEM solver variables.

\section{Methodology verification}

In this paper, the presented methodology is verified against the Amiet theory. For the BEM computations the commercial software LMS Virtual.Lab 11-SL1 is used. A systematic investigation regarding minimum mesh requirements and mesh convergence was conducted. In addition intermediate step verification against analytical results were also executed giving confidence to the authors about the present methodology. Figure 5 shows the polar plots for reduced wavenumbers $k c=1, k c=5$ and $k c=10$. These wavenumbers were chosen based on the fact that for $k c=1$ the region close to the airfoil leading-edge plays a major role in the noise generation. For this frequency, the airfoil is compact and can be considered as a dipole. As the frequency grows (e.g. $k c=5$ and $k c=10$ ) the compactness hypothesis becomes untrue and the airfoil loading is more distributed along the airfoil chord. Consequently, non-negligible constructive and destructive interferences between the noise sources occur, leading to the presence of side-lobes on the noise polar plot.

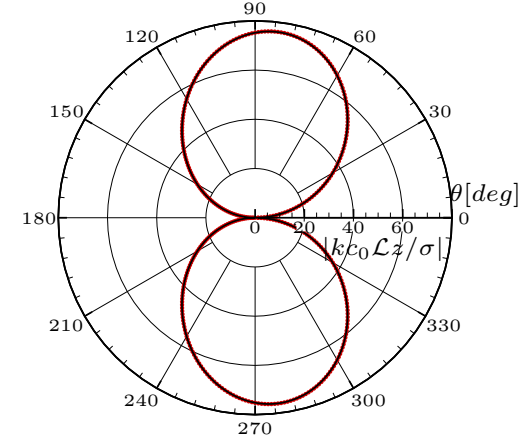

(a) $\mathrm{kc}=1$

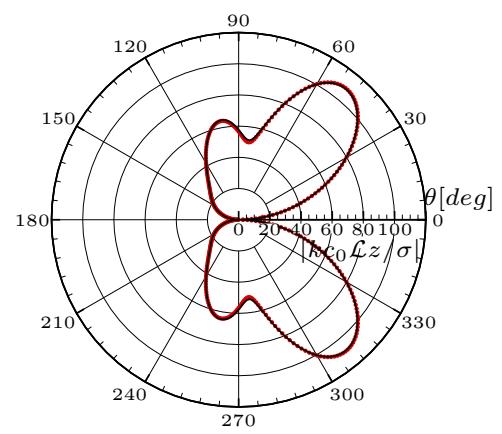

(b) $\mathrm{kc}=5$

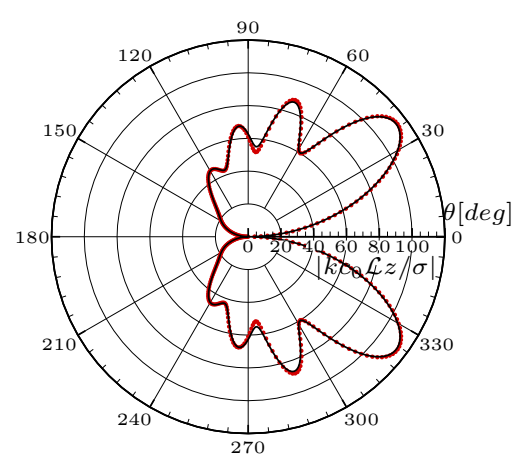

(c) $\mathrm{kc}=10$

Figure 5. Gust airfoil interaction noise polar for $k c=1, k c=5$ and $k c=10$. ( -

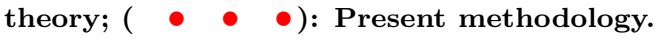

From fig. 5 it is seen that the present methodology gives excellent agreement for the $k c=1, k c=5$ and $k c=10$, i.e. up to highly non-compact conditions.

\section{Methodology application to a rotor-wake gust}

To study the applicability of the present methodology to practical cases, a wake-airfoil interaction problem was chosen. ${ }^{29}$ In this work an incoming wake profile is obtained from hot-wire measurements executed in the fan aeroacoustic facility of the von Karman Institute for Fluid Dynamics. ${ }^{32}$ This 3 blade rotor had its wake completely mapped at a distance of $0.01 \mathrm{~m}$ downstream of the trailing edge of the fan. This experimental facility allows setting the mass flow rate and pressure rise independently from each other, thanks to an auxiliary fan. For this mapping, the rotation speed of $1780 \mathrm{rpm}$ was set, for a bulk flow velocity of $15.15 \mathrm{~m} / \mathrm{s}$, the Blade Passing Frequency (BPF) is $87 \mathrm{~Hz}$. The experiments are discussed in full detail by De Santana. ${ }^{29}$ Figure 6 shows the velocity fluctuation around the mean value, averaged along the rotor radius, for one rotation.

Figure 7 shows the Power Spectral Density (PSD) of the velocity fluctuation in the direction normal to the mean flow.

From Fig. 7 it is noticed that, for the first harmonic, the velocity fluctuation amplitude is much larger than for the other harmonics. In addition, it is seen that, for this case, the second most important harmonic 


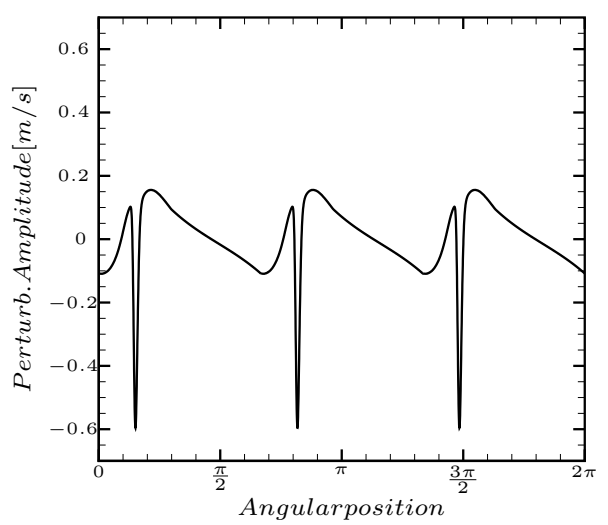

Figure 6. Wake gust velocity profile.

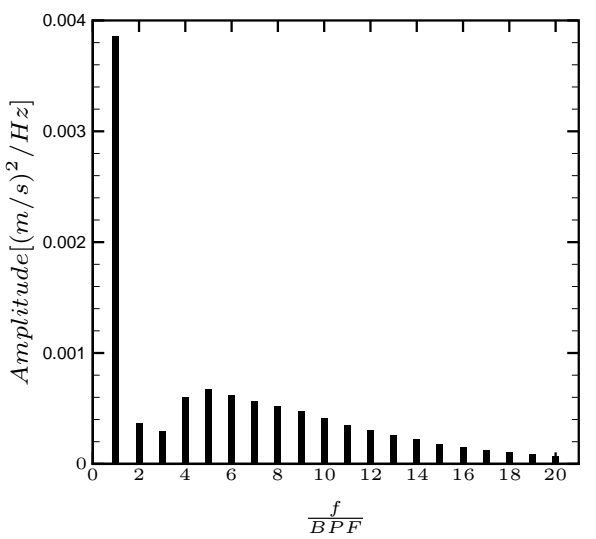

Figure 7. Upstream rotor downwash spectrum at the blade leading edge.

is the $5^{t h}$.

The characterized incoming flow profile was used as input for the present methodology to verify the applicability of the present technique to complex incoming flows. In this analysis the tonal response of the airfoil was tested for a flat plate (Amiet theory case) and for airfoils with $1 \%, 6 \%$ and $12 \%$ of thickness, with results shown in Fig. 8.

From Fig. 8 it is noticed that the thickness has an effect of noise reduction for all frequencies. In addition, it is seen that the noise reduction is more pronounced at higher frequencies. One interesting aspect to be noticed is that the amplitude relation of the predicted airfoil noise does not follow strictly the amplitude relation of the incoming gust. This fact is more remarkable for thick airfoils and shows the importance of taking into account more geometric details to increase the precision of the noise prediction. These results are qualitatively in agreement with the literature, ${ }^{2,8,12,15,18}$ showing that the present methodology presents consistent results. Further investigation is planned to be conducted to establish quantitative verification of this technique with experimental and CFD results.

\section{Conclusion}

An innovative technique for computing tonal noise at high frequencies applicable to blade-vortex-interaction, contra-rotating rotors and push propeller problems is presented. This numerically based methodology allows 


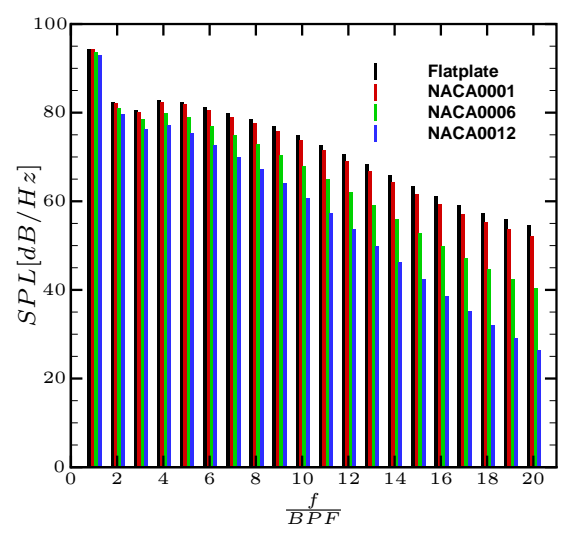

Figure 8. Acoustic spectrum comparison for the flat plate against thick airfoils.

accounting for airfoil geometrical effects and complex incoming flow profiles. In this methodology the small perturbations compressible linearized flow equations are rewritten as a Helmholtz equation which is solved by the Boundary Element Method. This technique has been verified against the analytical leading edge Amiet theory, showing excellent agreement at frequencies where the airfoil can be considered as compact noise source. As soon as the perturbation frequency grows, and the airfoil cannot be considered anymore a compact source, this technique shows small differences, in relation to the Amiet theory results, which can be overcome by increasing the mesh size and number of elements. Finally, the methodology of this work is applied to a case where the response of a flat plate and airfoils with $1 \%, 6 \%$ and $12 \%$ are computed and the predicted noise is presented. The results of these predictions are qualitatively in agreement with the literature, showing that the present methodology is a promising alternative for fast prediction of the generic airfoil response to complex incoming gust.

\section{Acknowledgments}

Leandro D. Santana acknowledges the Brazilian Coordination for Improvement of Higher Education Personnel - CAPES - process number BEX-0520-10-1. The authors gratefully acknowledge the support of the European Commission, in the framework of the FP7 project IDEALVENT (Grant Agreement no 314066). The IWT Flanders and the Fund for Scientific Research Flanders (F.W.O.) are also gratefully acknowledged for their support.

\section{References}

${ }^{1}$ Amiet, R., "Acoustic radiation from an airfoil in a turbulent stream," Journal of Sound and Vibration, Vol. 41, No. 4, 1975, pp. $407-420$.

${ }^{2}$ Paterson, R. W. and Amiet, R. K., "Noise and Surface Pressure Response of an Airfoil to Incident Turbulence," Journal of Aircraft, Vol. 14, No. 8, 1977, pp. 729-136.

${ }^{3}$ Atassi, H. M., "The Sears problem for a lifting airfoil revisited - new results," Journal of Fluid Mechanics, Vol. 141, 1984, pp. 109-122.

${ }^{4}$ Jacob, M. C., Boudet, J., Casalino, D., and Michard, M., "A rod-airfoil experiment as a benchmark for broadband noise modeling," Theoretical and Computational Fluid Dynamics, Vol. 19, 2005, pp. 171-196, 10.1007/s00162-004-0108-6.

${ }^{5}$ Mish, P. F. and Devenport, W. J., "An experimental investigation of unsteady surface pressure on an airfoil in turbulence Part 1: Effects of mean loading," Journal of Sound and Vibration, Vol. 296, No. 3, 2006, pp. 417 - 446.

${ }^{6}$ Mish, P. F. and Devenport, W. J., "An experimental investigation of unsteady surface pressure on an airfoil in turbulence Part 2: Sources and prediction of mean loading effects," Journal of Sound and Vibration, Vol. 296, No. 3, 2006, pp. 447 - 460.

${ }^{7}$ Staubs, J. K., Real Airfoil Effects on Leading Edge Noise, Ph.D. thesis, Virginia Polytechnic Institute and State University, 2008.

${ }^{8}$ Devenport, W. J., Staubs, J. K., and Glegg, S. A., "Sound radiation from real airfoils in turbulence," Journal of Sound and Vibration, Vol. 329, No. 17, 2010, pp. $3470-3483$.

${ }^{9}$ Lysak, P. D., Unsteady lift of thick airfoils in incompressible turbulent flow, Ph.D. thesis, The Pennsylvania State 
University, 2011.

${ }^{10}$ Roger, M., Schram, C., and Moreau, S., "On vortex-airfoil interaction noise including span-end effects, with application to open-rotor aeroacoustics," Journal of Sound and Vibration, Vol. Volume 333, 2013, pp. 283-306.

${ }^{11}$ Amiet, R., "Airfoil gust response and the sound produced by airfoil-vortex interaction," Journal of Sound and Vibration, Vol. 107, No. 3, 1986, pp. $487-506$

${ }^{12}$ Howe, M., "Correlation of lift and thickness noise sources in vortex-airfoil interaction," Journal of Sound and Vibration, Vol. 137, No. 1, 1990, pp. $1-7$.

${ }^{13}$ Howe, M., "Unsteady Lift And Sound Produced By An Airfoil In A Turbulent Boundary Layer," Journal of Fluids and Structures, Vol. 15, No. 2, 2001, pp. $207-225$

${ }^{14}$ Glegg, S. A. and Devenport, W., "Unsteady loading on an airfoil of arbitrary thickness," Journal of Sound and Vibration, Vol. 319, No. 3-5, 2009, pp. $1252-1270$.

${ }^{15}$ Glegg, S. A. and Devenport, W. J., "Panel methods for airfoils in turbulent flow," Journal of Sound and Vibration, Vol. 329, No. 18, 2010, pp. $3709-3720$

${ }^{16}$ Kucukcoskun, K., Christophe, J., and Tournour, C. S. M., "Broadband scattering of the turbulence-interaction noise of a stationary airfoil: experimental validation of a semi-analytical model," International Journal of Aeroacoustics, Vol. 12, 2013, pp. $1-12$.

${ }^{17}$ Roger, M. and Carazo, A., "Blade-Geometry Considerations in Analytical Gust-Airfoil Interaction Noise Models," $16^{\text {th }}$ AIAA/CEAS Aeroacoustics Conference, No. AIAA Paper 2010-3799, Stockholm, Sweden, 2010

${ }^{18}$ Moreau, S., Roger, M., and Jurdic, V., "Effect of Angle of Attack and Airfoil Shape on Turbulence-Interaction Noise," $11^{\text {th }}$ AIAA/CEAS Aeroacoustics Conference (26 ${ }^{\text {th }}$ AIAA Aeroacoustics Conference), No. AIAA 2005-2973, 2005.

${ }^{19}$ Gennaretti, M., Luceri, L., and Morino, L., "A Unified Boundary Integral Methodology For Aerodynamics And Aeroacoustics Of Rotors," Journal of Sound and Vibration, Vol. 200, No. 4, 1997, pp. $467-489$.

${ }^{20}$ Gennaretti, M., Testa, C., and Bernardini, G., "An unsteady aerodynamic formulation for efficient rotor tonal noise prediction," Journal of Sound and Vibration, Vol. 332, 2013, pp. 6743-6754.

${ }^{21}$ von Karman, T. and Sears, W., "Airfoil theory for non-uniform motion," Journal of the Aeronautical Sciences, Vol. 5, No. 10, 1938, pp. 379-390.

${ }^{22}$ Landahl, M. T., Unsteady Transonic Flow, Cambridge Science Classics, 1989.

${ }^{23}$ Osborne, C., "Unsteady thin-airfoil theory for subsonic flow," AIAA Journal, Vol. 11, 1973, pp. 205-209.

${ }^{24}$ Amiet, R. K., "Compressibility Effects in Unsteady Thin-Airfoil Theory," AIAA Journal, Vol. 12, 1974, pp. $252-255$.

${ }^{25}$ Adamczyk, J. J., "The Passage Of An Infinite Swept Airfoil Through An Oblique Gust," Tech. rep., National Aeronautics And Space Administration, 1974.

${ }^{26}$ Amiet, R. K., "High Frequency Thin-Airfoil Theory for Subsonic Flow," AIA Journal, Vol. 14, No. 8, 1976.

${ }^{27}$ Schwarzschild, K., "Die Beugung und Polarisation des Lichts durch einen Spalt. I," Mathematische Annalen, Vol. 55, No. 2, 1901, pp. 177-247.

${ }^{28}$ Roger, M. and Moreau, S., "Back-scattering correction and further extensions of Amiet's trailing-edge noise model. Part 1: theory," Journal of Sound and Vibration, Vol. 286, No. 3, 2005, pp. $477-506$.

${ }^{29}$ Santana, L. D., Schram, C., and Desmet, W., "An experimental procedure for the determination of wake-airfoil interaction noise parameters," $19^{\text {th }}$ AIAA/CEAS Aeroacoustics Conference, 2013

${ }^{30}$ Graham, J. M. R., "Similarity rules for thin aerofoils in non-stationary subsonic flows," Journal of Fluid Mechanics, Vol. 43, 1970, pp. $753-766$.

${ }^{31}$ Christophe, J., Application of Hybrid Methods to High Frequency Aeroacoustics, Ph.D. thesis, von Karman Institute for Fluid Dynamics, 2011.

${ }^{32}$ Bilka, M., Experimental investigation of rotor broadband self noise by means of rotating controlled diffusion blades, $\mathrm{Ph} . \mathrm{D}$. thesis, Vrije Universiteit Brussel, 2011. 